\title{
Conseqüências da violência familiar na saúde da criança e do adolescente: contribuições para a elaboração de propostas de ação
}

\author{
Consequences of family violence \\ to the health of children and adolescents: \\ contributions to action proposals
}

Michael E. Reichenheim 1

Maria Helena Hasselmann 2

Claudia Leite Moraes 1

\footnotetext{
1 Departamento de Epidemiologia do Instituto de Medicina Social e Núcleo de Pesquisa das Violências,

Universidade do Estado do Rio de Janeiro. Rua São Francisco Xavier, 524, 7o andar, bl. D, 20599-900 Rio de Janeiro, RJ, Brasil michael@ims.uerj.br.

2 Departamento de Nutrição Social do Instituto de Nutrição, da Universidade Federal do Rio de Janeiro e Núcleo de Pesquisa das Violências, Universidade do Estado do Rio de Janeiro
}

Abstract In Brazil, the magnitude of family violence is not yet fully known. Nevertheless, there is some evidence supporting that the issue should be immediately addressed. The article underlines the need for research, explicitly attempting to bridge the gap between generated knowledge and effective proposals for action. The article is divided in five parts. It opens with an overview concerning the importance of the problem of violence among children and adolescents. The following section presents a typology regarding main research lines on family violence. In the third section, one of those research lines is further detailed, especifically covering investigations dealing with the consequences of family violence. The conection between some of the findings and their use as subsidiaries for actual action proposals is addressed in the fourth section. The last section provides some comments about the importance of focusing violence at the family level as opposed to the individual level; the importance of handling the problem on a multi-professional basis; and the need to integrate agencies and services involved with family violence.

Key words Family Violence; Child Abuse; Violence against Adolescents; Prevention Strategies; Epidemiologic Studies
Resumo No Brasil, o conhecimento sobre a dimensão da violência familiar é ainda escasso. Todavia, existem evidências apontando para um cenário merecedor de enfrentamento imediato. Nesta perspectiva, o presente artigo destaca o campo das investigações sobre o tema, procurando explicitamente fazer uma ponte entre a apropriação do conhecimento gerado e efetivas propostas de ação. O artigo divide-se em cinco partes. Inicialmente, é discutida a importância da violência familiar no cenário de morbi-mortalidade de crianças e adolescentes A seguir, procura-se mapear as principais linhas de investigação sobre o tema, sugerindo uma tipologia de estudos da área. A terceira sessão explora com mais ênfase as investigações que avaliam as conseqüências do fenômeno na saúde de crianças e adolescentes. Na quarta seção, são discutidos os possíveis elos entre o conhecimento a ser gerado nesse âmbito e as propostas de ação para o enfrentando da violência familiar. $\mathrm{Na}$ última seção, são feitas algumas considerações sobre a importância do enfoque familiar em oposição ao individual; do multidisciplinar, em vez da abordagem compartimentalizada; e sobre a necessidade de integração das várias agências e serviços envolvidos com a violência familiar.

Palavras-chave Violência Familiar; Violência contra a Criança; Violência contra Adolescentes; Estratégias de Prevenção; Estudos Epidemiológicos 


\section{Introdução}

A partir da década de 70, a violência passou a ser uma das principais causas de morbi-mortalidade, principalmente na população de adolescentes e adultos jovens das grandes cidades (Minayo \& Souza, 1998). O incremento da participação relativa desses agravos no perfil de adoecimento e morte da população jovem tornou este grupo de causas um importante problema de saúde pública, intensificando e justificando uma centralização de esforços dos pesquisadores da área no sentido de conhecer melhor o problema. Até o momento, a magnitude das violências vem sendo avaliada pelas estatísticas de mortalidade por causas externas. Esta forma de abordagem das violências desvenda apenas a ponta de um grande iceberg, pois não contempla os casos não-fatais que, a cada dia, mais assumem um lugar de destaque nos quadros de morbidade de mulheres, crianças, adolescentes e adultos jovens. Pesquisadores da área de saúde sugerem que a violência interpessoal e as negligências que ocorrem no ambiente familiar sejam responsáveis por grande parte desses atos violentos (Minayo, 1994).

Apesar de a violência familiar ser descrita desde a antigüidade, somente há cerca de 30 anos é que o tema vem sendo sistematicamente discutido por pesquisadores da área de saúde (Straus \& Gelles, 1995; Gelles, 1997). A importância dada ao problema, não só no meio acadêmico como também na imprensa e no restante da sociedade civil, é conseqüência direta das estatísticas alarmantes encontradas ao longo dos últimos anos. Segundo Kashani et al. (1992), nos Estados Unidos, 4,1 milhões casos de violência familiar foram registrados no Departamento de Justiça, entre 1973 e 1981, configurando uma média anual de 450 mil casos. Estes números podem ser ainda maiores, pois refletem somente o comportamento das vítimas que registraram tais casos como criminais. Straus e Gelles (1995), após realização de dois inquéritos nacionais (1975 e 1985), também estimam uma alta incidência de violência nas famílias americanas. Apesar de terem notado um certo declínio nos índices entre os dois importantes estudos, os autores estimam que ainda cerca de 1.500 .000 crianças sejam vítimas de graves maus tratos e que cerca de 1.800.000 mulheres americanas sejam agredidas severamente por seus cônjuges, anualmente.
No Brasil, a magnitude da violência familiar ainda não pode ser bem dimensionada. $\mathrm{O}$ reconhecimento recente do problema, a utilização de diferentes definições do fenômeno pelas instituições e pesquisadores responsáveis pelas estatísticas disponíveis, a diversidade das fontes de informações existentes e a inexistência de inquéritos populacionais nacionais são alguns dos fatores que dificultam estimativas mais acuradas. Ademais, é importante reconhecer que a maioria das estimativas existentes na área reflete somente os casos mais visíveis e/ou graves de violência familiar. Possivelmente, a insuficiência de procedimentos básicos para o seu reconhecimento e de uma rotina clara e eficiente, capaz de estabelecer fluxos adequados das informações pertinentes entre as instituições envolvidas, também contribuem para o agravamento da situação.

Apesar das dificuldades apontadas, os resultados de algumas pesquisas nacionais indicam que a violência familiar no Brasil também é expressiva, devendo ser encarada como prioridade na agenda do Estado (Deslandes, 1997; Brasil, 1997). Segundo a Pesquisa Nacional por Amostra Domiciliar realizada no ano de 1988 (IBGE, 1989), 55\% das mulheres vítimas de agressão na Região Sudeste do Brasil haviam sido vitimizadas em seus próprios lares, sendo que, em $62,2 \%$ dos casos os agressores foram seus parentes ou conhecidos. No caso das crianças e adolescentes a proporção é ainda maior. Segundo a pesquisa, $80 \%$ das agressões físicas foram perpetradas por esses agressores.

No estado do Rio de Janeiro, estatísticas da polícia civil do ano de 1991 indicam que cerca de $70 \%$ dos homicídios de crianças de zero a onze anos foram perpetrados pela própria família (Soares, 1997). A magnitude da violência domiciliar também pôde ser evidenciada no inquérito realizado, durante os anos de 1990 e 1991, com escolares do município de Duque de Caxias. Nessa pesquisa, cerca de $33 \%$ das crianças e adolescentes entrevistados relataram a ocorrência de atos violentos na relação entre pais e filhos em seus domicílios (Assis, 1992).

Mesmo que ainda insuficientes, as estatísticas apresentadas acima apontam para um cenário merecedor de enfrentamento imediato. É fundamental a elaboração de propostas de ação com vistas à prevenção do problema e o acompanhamento de suas vítimas. Porém, se ações são indiscutivelmente prioritárias, o de- 
senvolvimento de linhas de investigação nessa área não deve ser deixado em segundo plano.

Nesta perspectiva, o presente artigo destaca o campo das investigações, procurando explicitamente fazer uma ponte entre a apropriação do conhecimento gerado e efetivas propostas de ação. Por mais que pareça lugar-comum afirmar a pesquisa como um dos fundamentos da ação social não se deve ignorar o fato de que muito do que se decide, e subseqüentemente se implementa, é pouco embasado em informações substantivas e, principalmente, sistematizadas. Se esta é uma crítica em geral, não seria um equívoco afirmar que no Brasil essa tem sido a regra, e não a exceção. Com o progressivo entendimento e conscientização da sociedade brasileira frente ao grave problema da violência familiar, passa a haver, ainda que reconhecidamente nos seus primórdios, uma crescente demanda por ações que a enfrentem. Por sua novidade, surge também uma excelente oportunidade de se criar um ambiente onde as políticas e ações sanitárias para o enfrentamento do problema passem a se articular intimamente a programas de investigação, não importando se dentro ou fora de academias.

Seguindo essa diretriz, o restante deste artigo divide-se em mais quatro partes. Na próxima seção, procura-se mapear as principais linhas de pesquisa em violência familiar no âmbito da Saúde Coletiva, oferecendo, mesmo que de forma tentativa e sujeita a debate e aprimoramentos, uma tipologia de estudos. A seção seguinte explora com mais ênfase um desses campos de estudo, especificamente o das pesquisas que avaliam as conseqüências diretas e indiretas do fenômeno na saúde de crianças e adolescentes. Procurando a ponte aludida acima, segue-se uma seção onde são discutidos os possíveis elos entre o conhecimento a ser gerado nesse âmbito e as possíveis propostas de prevenção da violência familiar. Finalmente, na última seção, são feitas algumas considerações sobre a importância do enfoque familiar em oposição ao individual; do multidisciplinar em vez da abordagem compartimentalizada; e sobre a necessidade de integração das várias agências e serviços envolvidos com a violência familiar.

\section{Uma tipologia dos estudos da área de violência familiar}

Iniciando uma descrição das linhas de investigação na área de violência familiar, vale ressaltar que a complexidade do fenômeno impõe que o conhecimento a ser produzido seja necessariamente construído por profissionais de diferentes áreas, destacando-se, entre outros, os profissionais de saúde, os cientistas sociais e os antropólogos. Embora reconhecendo que essa transdisciplinaridade implique vasto campo de estudo, dar-se-á ênfase aos estudos do âmbito direto da saúde coletiva.

Apresenta-se, a seguir, uma proposta de classificação dos estudos, agrupando-os conforme seus principais objetivos. A tipologia apresentada se baseia na leitura de títulos e resumos de cerca de 600 artigos publicados em periódicos indexados na rede Medline e Lilacs, no ano de 1996.

O primeiro grupo de investigações é composto por estudos interessados em discutir aspectos teórico-conceituais relacionados ao tema. Em geral, essas pesquisas são mais do âmbito dos cientistas sociais e antropólogos, por exemplo, aquelas enfocando as questões relacionadas à definição de violência familiar ou as que discutem as repercussões de questões eminentemente teóricas sobre o que Reichenheim e Moraes (1998) descrevem como validade operacional de investigações.

Um segundo grupo de trabalhos é constituído por estudos de freqüência (incidência, prevalência e freqüências relativas), que procuram dimensionar a magnitude da violência e identificar a sua importância com relação às demais violências, às outras causas externas e aos outros agravos em geral. Tradicionalmente, a epidemiologia é a disciplina que mais tem contribuído para o avanço do conhecimento na área.

O terceiro grupo se refere aos estudos descritivos que avaliam o perfil de vítimas e agressores, com a intenção de identificar grupos mais suscetíveis e vulneráveis. As informações geradas por essas investigações permitem identificar marcadores de risco, e contribuem para a elaboração de diagnósticos situacionais e para a identificação de subgrupos para ações sanitárias, incluindo a cobertura. As principais questões abordadas nesses estudos são as características espaço-temporais e pessoais envolvidas, bem como descrições das relações de parentesco entre vítimas e agressores. 
Uma quarta linha de pesquisa é representada pelos estudos sobre os possíveis determinantes/fatores de risco para a violência familiar. De acordo com a revisão bibliográfica realizada, os estudos abordam, principalmente, os fatores pessoais/psicológicos dos indivíduos envolvidos; a história de violência em gerações anteriores ou em idades mais precoces; fatores ambientais e sócio-econômico-culturais dessas famílias; e as características situacionais presentes nos momentos de violência familiar; estes últimos, os efetores mais diretos na ocorrência da violência.

Pesquisas aglutinando estudos que discutem questões relacionadas às propostas de ação para o enfrentamento do problema formam um quinto grupo. Essas investigações englobam a literatura sobre diretrizes, políticas e estratégias, globais ou locais, com vistas à redução da violência familiar. Pode-se incluir aqui também os estudos de avaliação das próprias estratégias. Tais pesquisas objetivam analisar os processos envolvidos nas ações ou a análise de resultados e impacto das ações implementadas. As pesquisas que avaliam a prática de profissionais de saúde na assistência às famílias vítimas de violência, ou as que exploram as relações entre as instituições envolvidas no atendimento das vítimas, são exemplos de estudos de processos. No outro conjunto, pode-se citar os estudos sobre mudanças nos conhecimentos, atitudes e práticas de profissionais de saúde após campanhas educativas sobre violência familiar, ou os que avaliam a redução da incidência ou prevalência de violência familiar após uma intervenção específica.

Uma sexta categoria tipológica é constituída pelos estudos que têm como objetivo construir e avaliar a validade e confiabilidade de instrumentos elaborados para a captação da violência familiar no nível empírico. Vale ressaltar que essas pesquisas se dedicam, prioritariamente, aos instrumentos de cunho quantitativo, capazes de rastrear as possíveis ocorrências de violência familiar em investigações epidemiológicas ou de casos.

Por fim, identifica-se um sétimo grupo de investigações, composto por estudos que abordam as conseqüências da violência familiar. Tais pesquisas analisam, entre outros, os custos sociais e econômicos, além de explorar as possíveis repercussões da violência no estado de saúde de suas principais vítimas. Este grupo de estudos é de especial interesse aqui, uma vez que a tipologia nos servirá para buscar os possíveis elos entre o conhecimento a ser gerado e as propostas de ação para o enfrentamento da violência familiar.

A próxima seção é dedicada a algumas das evidências já observadas nos estudos da área. São enfocadas, especificamente, as pesquisas sobre as conseqüências da violência na saúde da criança e do adolescente.

\section{Conseqüências da violência familiar na saúde da criança}

Os primeiros estudos na área abordavam, principalmente, as conseqüências traumáticas do abuso físico. Mais recentemente, este campo de investigação se ampliou e passou a incorporar um grande número de pesquisas que focalizam também as conseqüências psicológicas da violência familiar na saúde de suas vítimas (Kempe et al., 1962; Garbarino et al., 1988). No entanto, ainda são bastante escassas as investigações sobre o efeito indireto e complementar da violência familiar em processos de adoecimento mais complexos ou sobre os efeitos na saúde da criança em conseqüência do testemunho de violência entre outros membros da família. Um dos possíveis motivos para a carência de pesquisas desse tipo é a dificuldade de isolar o efeito da violência familiar em processos envolvendo fatores de risco relacionados entre si, nos quais o estabelecimento de hierarquia entre os mesmos é problemático.

Independentemente da forma de apresentação da violência, quer física, psicológica, sexual ou por negligência (Brasil, 1997), um expressivo número de autores aponta que as principais conseqüências dos maus-tratos na infância ocorrem no desenvolvimento infantil nas esferas física, social, comportamental, emocional e cognitiva (Kashani et al., 1992; Straus \& Gelles, 1995; Gelles, 1997). Essas investigações sugerem que a intensidade do problema depende da conjunção de vários fatores, tais como o desenvolvimento psicológico e a capacidade intelectual da criança; o vínculo afetivo entre o agressor e a vítima; a representação do abuso para a criança e a duração do mesmo; a natureza da agressão; ou ainda, as medidas em curso para a prevenção de abusos futuros.

Para facilitar a apresentação desta breve revisão, as conseqüências da violência familiar 
na saúde infantil podem ser classificadas por dois eixos. O primeiro discerne as conseqüências traumáticas (físicas), as emocionais ou afetivas, os agravos habitualmente explorados pelo domínio da saúde materno-infantil (desnutrição, baixo-peso ao nascer, etc.), e, por exclusão, as outras conseqüências que não se enquadram em nenhum dos grupos anteriores. O segundo eixo classifica as conseqüências com relação ao tempo decorrido entre a exposição à violência e o aparecimento do agravo. Neste sentido, as conseqüências podem ser imediatas, mediatas ou de longo prazo.

Vale ressaltar que, como qualquer esquema, esse também tem seus limites borrados, na medida em que tenta reduzir os conceitos às suas características mais marcantes e visíveis. Por vezes não se conhece o início do processo de instalação, o que dificulta a classificação do agravo com relação à sua temporalidade. Ademais, a associação entre os diferentes tipos de maus-tratos torna difícil a distinção da modalidade de abuso que gerou um certo agravo. A seguir, apresenta-se uma sintética descrição das conseqüências da violência familiar à saúde da criança.

\section{Conseqüências traumáticas/ agravos físicos}

As conseqüências da violência que diretamente atingem a saúde da criança ou do adolescente podem ser imediatas, de médio e longo prazo. As imediatas são mais facilmente identificadas, já que tendem a deixar marcas visíveis, principalmente na pele ou no sistema ósteo-articular. As conseqüências traumato-ortopédicas decorrentes de abuso físico, tais como traumatismos cranianos, luxações e fraturas e as lesões de pele como escoriações e hematomas, são os principais exemplos. Também não são raros os cortes, queimaduras e rompimento de órgãos (Abrapia, 1992; Hendricks-Matthews, 1993; Deslandes, 1997). Observa-se uma grande variação do espectro de gravidade desses agravos, tendo repercussões diretas sobre a notificação e a demanda de atenção médica. Na maioria das circunstâncias as lesões são leves e passam despercebidas; em alguns casos, os traumas são graves, necessitando de internação hospitalar, podendo levar inclusive ao óbito. Em geral, as situações mais graves são decorrentes de múltiplas lesões, habitualmente envolvendo a utilização de objetos, como cintos, pedaços de madeira e barra de ferro, entre outros.

Com relação aos abusos sexuais, deve-se destacar que a maioria ocorre sem que hajam quaisquer sinais físicos. Segundo relatos (Abrapia, 1992), em apenas uma pequena parcela de casos é possível identificar lesões físicas que demonstrem a ocorrência desse tipo de abuso. Conspicuamente, muitos abusos sexuais se dão sem que haja qualquer violência física ou vestígios corporais e são geralmente perpetrados repetidamente por alguém conhecido da vítima. Algumas vezes ocorrem conseqüências físicas locais imediatas, como lesões himenais, escoriações, hematomas locais, laceração de períneo, além de traumas em outras regiões corporais, em decorrência de abuso físico simultâneo (Rappley \& Speare, 1993; Levitt et al., 1993)

As conseqüências de situações de negligência são mais difíceis de se caracterizar e, portanto, de se identificar. Isto é ainda mais complicado em sociedades nas quais importante parcela das crianças e adolescentes são vítimas crônicas do descuido do próprio Estado, que não lhes garante condições mínimas necessárias ao crescimento e desenvolvimento. Nestas circunstâncias, a própria definição de negligência familiar é pouco clara, o que dificulta a imputação de causalidade em certas associações encontradas. Apesar disso, é possível postular que crianças negligenciadas pela família e pouco supervisionadas corram maior risco de ser vítimas de "acidentes domésticos" do que crianças não negligenciadas. Sob este rótulo encontram-se as quedas, os envenenamentos, as queimaduras graves ou mesmo os atropelamentos peridomiciliares. As conseqüências podem ser as mais variadas, desde simples escoriações geradas por traumas leves, até a própria morte da criança ou adolescente.

\section{Conseqüências emocionais/ agravos afetivos}

Sendo geralmente de médio e longo prazos, as conseqüências emocionais são também de identificação difícil. A gama de resultados do abuso emocional, físico e sexual continuados é vasta. Autores relatam distúrbios psicossomáticos gastrointestinais crônicos e remitentes, ou dores abdominais inespecíficas; repercussões psicoemocionais, como a ansiedade 
ou a depressão; dificuldade de relacionamento e comportamento manifestada por agressividade, timidez, isolamento social progressivo e distúrbios do sono e do apetite; ou ainda, problemas na esfera de atividades, como por exemplo a baixa performance social e intelectual (Kashani et al., 1992; Hendricks-Matthews, 1993; Straus \& Gelles, 1995; Gelles, 1997).

Algumas pesquisas sugerem também que a punição corporal na adolescência possa estar associada ao quadro de abuso de álcool e drogas, depressão e tentativas de suicídio na idade adulta (National Research Council, 1993 apud Gelles, 1997). Recentemente, estudos vêm apontando uma relação entre história de violência familiar na infância e criminalidade na adolescência (Widom, 1989; Aber et al., 1990). Em vítimas de abuso sexual, também é descrita uma inter-relação dos agravos mencionados acima com comportamentos sexuais incompatíveis com os esperados para a idade (Kendall-Tackett et al., 1993).

Um outro enfoque, que somente nas últimas duas décadas tem recebido alguma ênfase, concerne às possíveis conseqüências, para a saúde da criança, do testemunho da violência entre os pais (Freidrich \& Einbender, 1983; Kazdin et al., 1985). Alguns autores apontam que, diferentemente do que se supunha, as conseqüências emocionais da convivência das crianças em situações de grande conflito podem ser até mesmo piores do que quando elas mesmas são o alvo de violência. Tal como nos casos de violência diretamente infligida, estudos sugerem a ocorrência de distúrbios ulteriores nos níveis emocionais, cognitivos e de comportamento. A percepção de que vivem em famílias sem limites, conflituosas e perigosas, misturando-se à freqüente culpabilidade que carregam da violência entre os pais, é parte do cenário de desencadeamento de agravos psicoemocionais, quer de curto, médio ou longo prazos (Layzer et al., 1985; Hughes, 1988; Jaffe et al., 1990).

Fato que merece atenção especial é que a violência entre membros do casal também pode vir acompanhada da violência específica contra a criança. Isto torna difícil compreender o efeito isolado de cada fator contribuinte no processo de ocorrência desses problemas (Hilberman, 1980). Algumas pesquisas têm mostrado que essa concomitância tem efeito sinérgico sobre a saúde da criança e que a gravidade da situação conjunta é maior que a soma dos efeitos dos dois abusos em separado (Kashani et al., 1992).

\section{Agravos habitualmente explorados no domínio da saúde materno-infantil}

Como mencionado no início da seção, ainda são escassas as linhas de investigação que se debruçam sobre a violência familiar como um dos fatores de risco para os agravos classicamente estudados no domínio materno-infantil (desnutrição, baixo-peso ao nascer, infecções no primeiro ano de vida, etc.). Descrevem-se, a seguir, algumas evidências que têm emergido nos últimos anos e que, pela falta de consenso e precocidade de seus programas de investigação, merecem ser mais bem estudadas.

\section{Violência familiar e estado nutricional}

Poucos estudos existem sobre as possíveis conseqüências da violência familiar na situação nutricional infantil. Assumindo que um ambiente familiar conflituoso e hostil pode levar a uma situação de estresse importante para toda a família, alguns autores (Karp et al., 1989; Valdez-Santiago \& Sanín-Aguirre, 1996) vêm apontando para uma possível associação entre violência familiar e deficiências nutricionais, quer intra-uterinas, quer nos primeiros anos de vida.

Na maioria, esses artigos são investigações transversais, o que apenas sugerem a existência de associação entre a ocorrência de maustratos e a desnutrição (Bullard et al., 1967; Birrel \& Birrel, 1968; Bernard \& Wolf, 1973; Martin, 1973; Krieger, 1974; Martin et al., 1974). As pesquisas descrevem condições denominadas failure to thrive e dwarfism, que assinalam a falha do crescimento por determinações sociais e psicológicas (Bullard et al.. 1967; Birrell \& Birrel, 1968; Martin et al., 1974). Segundo estudo realizado pelo Denver Department of Welfare, a existência de um ambiente familiar ofensivo é um elemento tão ou mais importante para o desenvolvimento da criança que o abuso em si. Questiona-se se esse ambiente ofensivo não poderia estar contribuindo também para a ocorrência de problemas nutricionais (Martin et al., 1974; Battacharyya, 1983). Segundo os autores, o abuso físico da criança usualmente ocorre em associação com algumas outras condições ambientais desfavoráveis. A negligência nutricional, o abuso sexual, a presença de agravos emocionais severos em um ou ambos os pais e a privação 
econômica e social são situações freqüentemente concorrentes em famílias que maltratam suas crianças. Desta forma, uma situação nutricional inadequada pode estar associada a qualquer um desses fatores, isoladamente ou em conjunto com os demais.

Outra questão a ser considerada é a relação psicológica existente entre mãe e filho no processo de determinação do crescimento infantil. Alguns autores abordam o tema estudando um quadro complexo conhecido como "síndrome de privação materna" (Whitten et al., 1969). Essas pesquisas sugerem uma relação entre os cuidados com a criança, o estado psicológico e emocional da mãe e a falta de crescimento físico na infância. São sugeridos dois processos básicos para a ocorrência de agravos nutricionais em crianças submetidas a tal situação. No primeiro, e mais direto, a desnutrição ocorreria como resultado do não oferecimento e aporte inadequado de alimentação. No segundo, a desnutrição seria o elo final de um processo envolvendo primeiramente a ocorrência de problemas emocionais importantes levando, subseqüentemente, à diminuição de apetite e a uma progressiva e sistemática recusa alimentar.

\section{Violência na gestação e agravos no recém-nascido}

Ainda existem poucos estudos epidemiológicos com o objetivo de discutir as conseqüências da violência durante a gestação na saúde da gestante e da criança. As poucas evidências, no entanto, apontam certos perfis que merecem aprofundamento. Alguns estudos indicam que as gestantes vitimizadas iniciam o pré-natal mais tardiamente, o que dificultaria a identificação de comportamentos de risco, como tabagismo, uso de medicamentos proscritos ou drogas ilícitas durante a gestação, e patologias pregressas ou correlatas à gravidez, acarretando um possível aumento de complicações maternas e fetais (Hillard, 1985; Stewart \& Cecutti, 1993). As pesquisas que estudam as conseqüências na saúde do feto sugerem a possibilidade de associação do abuso físico na gestação com várias conseqüências deletérias, como o aborto espontâneo ou hemorragias intracranianas com conseqüente morte fetal. Também são citados o descolamento prematuro de placenta, que aumentaria o risco de prematuridade e outras complicações maternas e fetais (Pugh, 1978; Goodwin \& Breen, 1990; Berenson et al., 1994).

Recentemente, investigações vêm apontando uma possível associação entre a violência contra a mulher durante a gestação e o baixopeso ao nascer (Bullock \& McFarlane, 1989; Schei et al., 1991; Parker et al., 1994 e ValdezSantiago \& Sanín-Aguirre, 1996). Os resultados conflitantes e os sérios problemas metodológicos encontrados nessas investigações indicam a necessidade de novos estudos sobre o tema. A maioria das pesquisas utiliza delineamentos inadequados e tem pouco cuidado com os rigores de definição, desenho e execução necessários a interpretações causais. Outra questão importante é que a maioria das pesquisas foi realizada nos Estados Unidos; apenas três estudos são provenientes de outros países.

Vale enfatizar a pertinência dessas investigações face às lacunas que ainda existem nos programas de investigação sobre os determinantes do baixo-peso ao nascer e de seus principais componentes (prematuridade e crescimento intra-uterino retardado). As pesquisas na área têm destacado que tais agravos são originados por um conjunto de dimensões interrelacionadas e apenas parcialmente conhecidas. Nesse debate, muito se tem questionado sobre o efeito de fatores psicossociais maternos na gênese dos desfechos. O grande número de investigações na área é decorrente da inconsistência dos achados anteriores. Parece pertinente sugerir que um ambiente familiar violento durante a gestação possa levar a uma situação conflituosa importante para toda a família, especialmente para a gestante que, por questões próprias da gravidez, é mais suscetível a qualquer evento estressante. Desta forma, torna-se interessante acoplar a estes programas de investigação pesquisas que discutam as possíveis repercussões da violência durante a gestação no desenvolvimento intrauterino da criança.

\section{Outras conseqüências}

As investigações que abordam as conseqüências do fenômeno na saúde das mulheres relatam que aquelas vitimizadas por pessoas do círculo próximo de relações em seus próprios domicílios estão mais sujeitas a uma série de sintomas e formas de adoecimento (Hilberman, 1980; Giffin, 1994; Heise, 1994). Os pro- 
blemas de saúde vão desde dificuldades adaptativas até a própria morte. São citados o isolamento social, a insegurança, a depressão, os distúrbios do sono, a baixa auto-estima, a presença de dores abdominais recorrentes, lombalgias crônicas, cefaléia, contusões localizadas e distúrbios psicossomáticos (Heise, 1994; Minayo, 1994; Valdez-Santiago \& Sanín-Aguirre, 1996). Outras investigações têm indicado uma importante associação entre o fenômeno e o uso de álcool, fumo e drogas ilícitas (Mc Farlane et al., 1996; Martin et al., 1996; Gelles, 1997). O estresse permanente, a decepção com o companheiro e a falta de esperança de modificação da situação de violência são os principais precursores da aquisição ou intensificação desses hábitos de vida (Giffin, 1994).

Observando-se a variada gama de conseqüências da violência familiar na saúde das mulheres que vivenciam essa experiência, é possível questionar sua plena capacidade de educar e criar as crianças de forma adequada. Como será discutido nas considerações finais do artigo, apesar de a possibilidade da violência familiar vitimizar diretamente apenas um integrante da família, indiretamente, seus efeitos são observados em todos os membros do núcleo familiar. Possivelmente, tais repercussões são preferencialmente observadas nas crianças menores, em função do conhecimento existente sobre as estreitas relações entre o estado de saúde físico e mental materno e o crescimento e desenvolvimento infantil nos primeiros anos de vida (Beautrais et al., 1982; Fergusson et al., 1984; Freeman, 1984).

Também merecem menção algumas conseqüências da negligência não abordadas anteriormente: os atrasos ou falha completa no esquema de vacinação, que aumentam o risco das doenças imunopreviníveis; os hábitos higiênicos inadequados, que aumentam o risco de doenças diarreicas, dermatológicas ou bucais; e a falta de adesão a prescrições ou ausência de tratamento adequado pelos pais, levando ao agravamento da doença em questão (Abrapia, 1992).

Outro ponto que tem sido discutido concerne às conseqüências de estupros e outros abusos sexuais. Além da gravidez, quase sempre indesejada, merece menção o potencial risco de se contrair doenças sexualmente transmissíveis, destacando-se a infecção pelo HIV. Estudos apontam para um risco aumentado em contrair infecções, o que vem justificando a utilização de tratamento profilático para algumas dessas patologias em instituições de referência nacionais para o atendimento de adolescentes e mulheres vítimas de violência sexual (Santos, 1991; Levitt, 1993).

Também vem sendo debatido intensamente o papel da violência durante a infância e a adolescência na reprodução da violência familiar (Belsky, 1993). Um dos principais fatores de risco para a ocorrência dessa violência é a história pregressa de brutalidade durante a infância, em um ou mais membros da família. Apesar de não ser consensual, a hipótese de transmissão entre gerações tem um grande número de adeptos entre os pesquisadores da área. Segundo estudos norte-americanos, as taxas de abuso de mulheres é mil vezes maior em homens que presenciaram violência familiar em suas infâncias do que nos que não a presenciaram (Straus et al., 1980). Além disso, alguns autores que trabalham com mulheres vítimas de violência indicam uma maior dificuldade nas mulheres que presenciaram violência grave entre os pais para romper o ciclo da violência e se afastarem dos maridos agressores (Jaffe et al., 1990). Segundo Belsky (1993), os opositores à idéia de transmissão da violência entre gerações se baseiam nas críticas metodológicas cabíveis na maioria dos estudos sobre o tema. Tais críticas envolvem o reduzido tamanho amostral das pesquisas; a excessiva confiança depositada em informações retrospectivas; o grande número de pesquisas sem um grupo controle ou com controles inadequados; e o possível viés de informação decorrente do conhecimento da atual situação de agressor nos estudos que avaliam a ocorrência de violência na infância, através de entrevistas não estruturadas.

\section{Elo entre as informações geradas por investigações sobre as conseqüências da violência familiar e ações sanitárias}

Ao longo do texto foram descritas as linhas de investigação sobre violência familiar no âmbito da saúde coletiva e uma atenção especial foi dada aos estudos interessados em suas conseqüências na saúde da criança e do adolescente. Mesmo levando em conta que muitas dessas informações ainda são precárias e insuficientes, é sempre interessante procurar entender a conexão entre um possível conhecimento e as práticas de saúde que dele se bene- 
ficiam. Esta seção visa a chamar a atenção para a questão.

Na Figura 1, propõe-se um modelo esquemático das fases do processo de atenção à violência familiar. O intuito do modelo é facilitar o entendimento dos exemplos oferecidos adiante sobre as possíveis articulações entre o conhecimento gerado pelas investigações a respeito das conseqüências da violência familiar e as ações relacionadas. Uma idéia geral dos processos que envolvem uma ação de saúde permite a identificação dos atores envolvidos em cada etapa, de suas possíveis atribuições e tarefas e, não menos, permite apontar o conhecimento necessário para que esses atores decidam e executem melhor suas ações.

O modelo se restringe propositadamente ao âmbito do setor saúde. No entanto, é fundamental entender que qualquer ação a ser tomada nesse campo se aninha intimamente na esfera das decisões macrossociais e políticas, sem as quais não há norte. Na perspectiva da pesquisa, vale a pena enfatizar a importância do desenvolvimento de estudos que discutam e articulem conceitos habitualmente pesquisados pelas ciências sociais, como o conceito de justiça social, de cidadania, de solidariedade e de reciprocidade com a violência familiar. As relações entre essas questões, bem como o papel dos meios de comunicação de mas- sa e o crescimento da violência nos últimos anos, vêm sendo discutidos por vários autores da área (McRobbie \& Thornton, 1995; Velho, 1996; Wieviorka, 1997; Núcleo de Pesquisa das Violências, 1998). No entanto, no âmbito precípuo da violência na família, os estudos são escassos e devem ser encorajados.

Como sugere a Figura 1, as estratégias de ação sanitária no âmbito da violência familiar podem ser encadeadas em três etapas distintas. A de prevenção primária envolve, por exemplo, a incorporação de atividades de educação em saúde às rotinas dos serviços; as atitudes e comportamentos frente aos conflitos familiares; a importância de a violência familiar tornar-se pública; as possíveis apresentações da violência e suas principais características; as informações sobre locais de atendimento a vítimas de violência familiar; e a importância da notificação são alguns dos pontos que poderiam ser trabalhados.

Em termos de detecção, pode-se identificar dois momentos distintos: as ações precoces de detecção se referem a uma rede de captação, envolvendo instâncias e atores particulares. Aqui são importantes os fluxos de informação, especialmente os sistemas de notificação a partir de denúncias e eventos sentinela. O segundo momento enfatiza o processo da investigação da suspeita, passando pelo apro-

\section{Figura 1}

Modelo esquemático das ações relativas à violência familiar no âmbito da Saúde Coletiva.

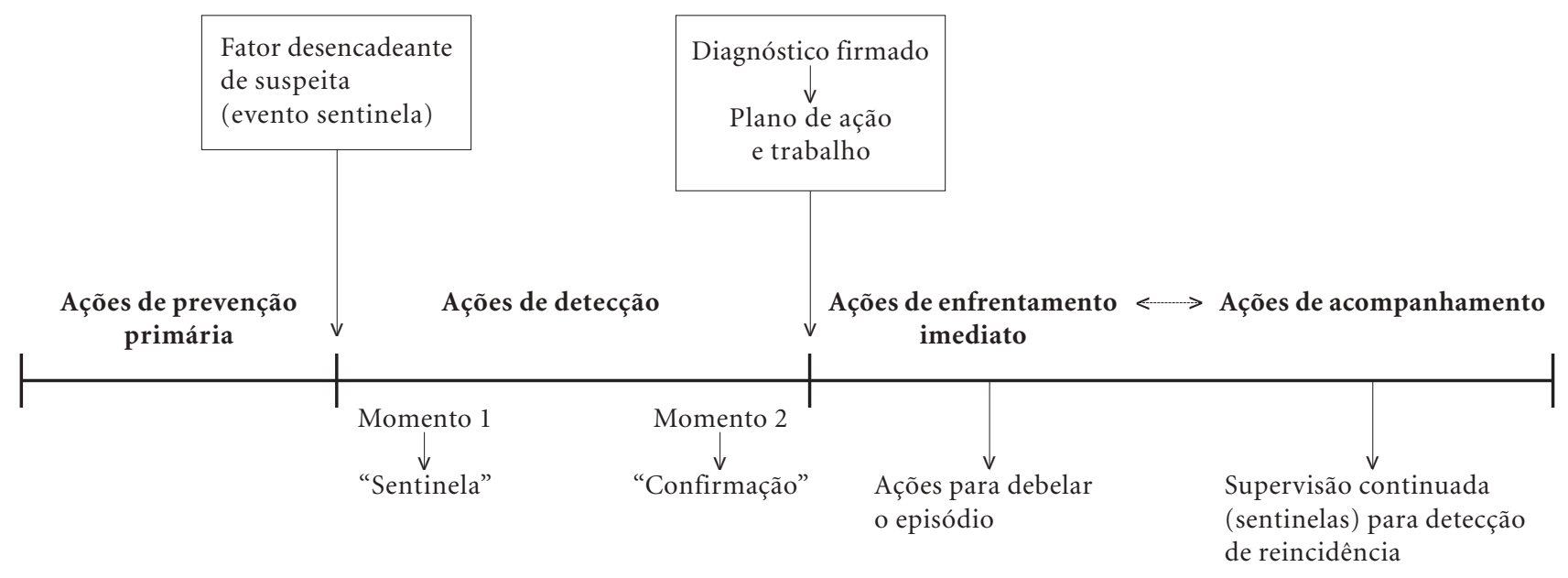


fundamento e discussão do caso por equipes multiprofissionais e culminando em uma eventual confirmação (ou refutação) de violência na família.

Uma vez constatada e confirmada uma situação de violência, a terceira etapa inicia-se com a formulação de um plano de ação envolvendo os integrantes da família, os profissionais do serviço e, possivelmente, de outras instituições de referência (por exemplo, abrigos temporários, o poder judiciário, etc). Dependendo da gravidade e particularidade da situação, pode-se visualizar duas formas de enfrentamento, não mutuamente exclusivas. Uma concerne a questões agudas que necessitam ser debeladas imediatamente, envolvendo medidas emergenciais na assistência à vítima, notificação aos órgãos competentes para devidas providências, etc. Pressupondo um controle da situação emergencial, a outra forma de atuação envolve o acompanhamento da família, visando à detecção do afloramento de novas situações de conflito; ações familiares de longo prazo, como, por exemplo, participação em grupos de auto-ajuda; ou ainda ações de ajuda ao nível individual. Como indica a seta pontilhada na Figura 1, havendo uma "agudização” da situação de conflito na família, ações emergenciais são reativadas.

Com esse esquema em mente, pode-se buscar os momentos em que as informações provenientes das pesquisas que abordam as conseqüências na saúde são oportunas para embasar decisões apropriadas. Em relação aos traumas físicos, o conhecimento de suas manifestações mais freqüentes teria repercussão direta na suspeição de casos pelos serviços de saúde, especialmente os de emergência. A ocorrência de lesões não explicadas, a contento, pelos familiares, sugere que a possibilidade de a criança ter sido vítima de uma lesão intencional por algum membro da família deva ser investigada pelos profissionais que atendem o caso. Detalhes sobre as conseqüências psicoemocionais, como depressão, ansiedade, distúrbios neurovegetativos e do sono são importantes, tanto para a especificidade das ações terapêuticas no período de acompanhamento, quanto para a suspeição de problemas recrudescentes entre os membros da família.

Entender os elos entre a violência e os agravos no âmbito materno-infantil pode ser extremamente interessante, não só para o enfrentamento dos agravos per se, como na eleição destes como eventos-sentinela para a si- tuação de violência. O conhecimento de que existe uma criança severamente desnutrida na família, ou da ocorrência de baixo-peso sem outros fatores clínicos que o expliquem, pode ser útil tanto numa fase inicial de detecção, quanto na fase de acompanhamento, apontando para possíveis recrudescimentos de conflitos marcantes. Profissionais de saúde certamente se beneficiariam do aumento na capacidade de identificar precocemente casos de violência nos serviços, acelerando as ações pertinentes.

Além das possibilidades de atuação direta, a expansão do conhecimento sobre a vasta gama de conseqüências da violência, bem como sobre a gravidade de suas manifestações, também se justifica pelo importante papel que pode ter em termos de sensibilização e engajamento de profissionais de saúde nos programas de prevenção, diagnóstico precoce e acompanhamento. Além disso, a própria consideração da necessidade de enfrentamento imediato do problema pelos planejadores de políticas públicas do setor saúde também será facilitada pela aquisição e divulgação dos resultados das investigações. O conhecimento sobre a diversidade dos agravos pode servir para colocar definitivamente a violência familiar nas agendas de atuação do setor.

\section{Considerações finais}

Percorrendo as questões abordadas ao longo deste texto, parece crucial entender a violência familiar como um fenômeno complexo que envolve todos os integrantes do núcleo familiar e que não se restringe a um indivíduo ou uma relação específica. Considerando as repercussões na saúde da mulher apontadas acima, é fácil imaginar que, mesmo confinadas ao casal, as conseqüências da violência se darão sobre toda a família. Como exigir que uma mãe que passa por essas experiências possa cuidar adequadamente de seus filhos? Como evitar que as crianças sejam negligenciadas ou mesmo agredidas física e emocionalmente? Gelles (1997) alerta para o fato de que, a despeito das reconhecidas peculiaridades das diversas modalidades de violência - violência contra a mulher, violência contra a criança, abuso infantil, abuso de idosos, entre outras - a análise isolada dos fenômenos não contribui para um melhor conhecimento e enfrentamento do problema. 
A abordagem familiar da violência e a própria complexidade do fenômeno traz como conseqüência a necessidade de integrar diferentes profissionais através da formação de equipes interdisciplinares em qualquer programa de prevenção, detecção e acompanhamento de vítimas. Ressalta-se a importância da permanente discussão dos casos atendidos por todos os integrantes da equipe responsável pelo acompanhamento da família. As especificidades de cada profissional permitem que a situação seja discutida de diferentes perspectivas, facilitando a confirmação do evento e o planejamento das atividades a serem desenvolvidas.

Neste sentido, esforços merecem ser dirigidos visando a quebrar efetivamente barreiras disciplinares, fazendo com que essas equipes não sejam meros retalhos, integradas por profissionais primordialmente alocados nos seus tradicionais setores (pediatria, psicologia, enfermagem, serviços sociais, etc.) e, despojadamente, participando das atividades nas "horas-extras". Ao contrário, essas equipes interdisciplinares deveriam ser compostas por profissionais dedicados em tempo integral, permitindo concentração de esforços e expertises. Para colocar essa proposição em perspectiva, vale perguntar se um agravo que atinge milhares, senão milhões, de crianças, adolescentes e mulheres, não merece um "setor" específico.

O engajamento de programas que tenham a família como alvo de intervenção nas estratégias de ação também deve ser considerado, visto que as atividades realizadas tendem a estreitar as relações entre o serviço de saúde e a comunidade; facilitar a identificação de famílias de risco (adolescentes grávidas e famílias onde haja abuso de álcool e drogas ilícitas, por exemplo); possibilitar o levantamento das possíveis redes sociais de apoio disponíveis; e permitir uma frutífera prática interdisciplinar de profissionais envolvidos com o atendimento das famílias.

Outro nível de integração necessário se dá entre as diferentes instituições envolvidas na prevenção da violência. Atualmente, ainda se observa uma real dificuldade de trabalho conjunto e retroalimentado entre organizações (setor judiciário, conselhos tutelares, organizações não-governamentais, etc). A divulgação e integração das atividades realizadas, o retorno de informações sobre o andamento dos casos e a especificação de ações, evitando a sobreposição de serviços, ainda são metas a serem atingidas. Vale ressaltar a insuficiência de programas de avaliação dos processos implementados, alicerce fundamental para o aumento da efetividade das ações realizadas.

Como alerta final, não se deve perder a perspectiva da escassez de informações existentes sobre a violência familiar. Que o panorama oferecido acima não crie a ilusão de substância. Muito do que foi exposto ainda demanda aprofundamento. Porém, no afã de atuar, há uma tendência de se aceitar conjecturas ou evidências ainda tênues para o embasamento de decisões e ações. Isto requer atenção. Para enfrentar a questão, há uma premência de discussões sobre as prioridades e rumos de programas de investigação na área, identificando as lacunas existentes a cada passo de cada fase de atuação.

\section{Referências}

Aber JL, Allen JP, Carlson V \& Cicchetti D 1990. The effects of maltreatment on development during early childhood: recent studies and their theoretical, clinical and policy implications, p. 579-619. In D Cicchetti \& V Carlson (orgs.) - Child Maltreatment: Theory and Research on Causes and Consequences. Cambridge University Press, New York.

Abrapia 1992. Maus Tratos contra Crianças e Adolescentes: Proteção e Prevenção - Guia de Orientação para Profissionais de Saúde. Abrapia, Petrópolis, 32 pp.

Assis SG 1992. Violência, Criança e Comportamento. Um Estudo em Grupos Sociais Distintos. Relatório final de pesquisa. Centro Latino Americano de Estudos Sobre Violência e Saúde/Fundação Oswaldo Cruz, Rio de Janeiro, (mimeo). 
Battacharyya AK 1983. Child abuse and neglect (CAN): Indian perspective. Indian Academy of Pediatrics 20: 803-810.

Beautrais AL, Fergusson DM \& Shannon FT 1982. Life events and childhood morbidity: a prospective study. Pediatrics 70: 935-940.

Belsky J 1993. Etiology of child maltreatment: a developmental-ecological analysis. Psychological Bulletin 114: 413-434.

Berenson AB, Wiemann CM, Wilkinson GS, Jones WA \& Anderson GD 1994. Perinatal morbidity associated with violence experienced by pregnant women. American Journal of Obstetrics and Gynecology 170: 1760-1769.

Bernard MV \& Wolf L 1973. Psychosocial failure to thrive. Nursing Clinics of North America 8: 557-565.

Birrell GR \& Birrell JHW 1968. The maltreatment syndrome in children: hospital survey. The Medical Journal of Australia 2: 1023-1029.

Brasil 1997. Violência contra a Criança e o Adolescente: Proposta Preliminar de Prevenção e Assistência à Violência Doméstica. Ministério da Saúde/SASA, Brasília. $24 \mathrm{pp}$.

Bullard DM, Galser HH, Hegarty MC \& Pivchik EC 1967. Failure to thrive in the "neglect" child. American Journal of Orthopsychiatry 37: 680-690.

Bullock L \& Mc Farlane J 1989. The birth weight battering connection. American Journal of Nursing 89: 1153-1155.

Deslandes SF 1997. Prevenir a Violência: um Desafio para Profissionais de Saúde. Fundação Oswaldo Cruz/Escola Nacional de Saúde Pública/Centro Latino-Americano de Estudos Sobre Violência e Saúde Jorge Careli, Rio de Janeiro, 39 pp.

Fergusson DM, Horwood LJ \& Shannon FT 1984. Relationship of family life events, maternal depression and child-rearing problems. Pediatrics 75: 30-35.

Freeman HL 1984. The scientific background. In HL Freeman, Mental Health and the Enviroment. Churchil-Livingstone, London.

Friedrich WN \& Einbender AJ 1983. The abused child: a psychological review. Journal of Clinical Child Psychology 12: 244-256.

Garbarino J, Guttmann E \& Seeley JW 1988. The Psychologically Battered Child. Jossey-Bass, London, 249 pp.

Gelles RJ 1997. Intimate Violence in Families. SAGE Publications, London, $188 \mathrm{pp}$.

Giffin K 1994. Violência de gênero, sexualidade e saúde. Cadernos de Saúde Pública 10: 146-155.

Goodwin TM \& Breen MT 1990. Pregnancy outcoming and fetal-maternal hemorrhage after noncatastrophic trauma. American Journal Obstetrics and Gynecology 162: 665-671.

Heise L 1994. Gender-based abuse: the global epidemic. Cadernos de Saúde Pública 10: 135-145.

Hendricks-Matthews MK 1993. Survivors of abuse: health care issues. Primary Care 20: 391-406.

Hilberman MD 1980. Overview: the "wife-beater's wife" reconsidered. American Journal of Psychiatry 137: 1336-1347.

Hillard PJA 1985. Physical abuse in pregnancy. Obstetrics \& Gynecology 66: 185-190.

Hughes HM 1988. Psychological and behavior correlates of family violence in child witnesses and victims. American Journal Orthopsychiatry 58: 77-90.
IBGE - Fundação Instituto Brasileiro de Geografia e Estatística 1989. Crianças e Adolescentes: Indicadores Sociais. IBGE, Rio de Janeiro.

Jaffe PG, Hurley DJ \& Wolfe D 1990. Children's observations of violence: I. Critical issues in child development and intervention planning. Canadian Journal of Psychiatry 35: 466-470.

Karp RJ, Scholl TO, Decker E \& Ebert E 1989. Growth of abused children. Contrasted with the non-abuses in an urban poor community. Clinical Pediatrics 28: 317-320.

Kashani JH, Daniel AE \& Dandoy AC 1992. Family violence: impact on children. Journal of the American Academy of Child and Adolescent Psychiatry 31: 181189.

Kazdin AE, Moser J \& Colbus D 1985. Depressive symptoms among physically abused and psychiatrically disturbed children. Journal of Abnormal Psychology 94: 298-307.

Kempe C, Silverman F, Steele B, Droegemueller W \& Silver H 1962. The battered child syndrome. Journal of American Medical Association 181: 107-112.

Kendall-Tackett KA, Williams L \& Finkelhor D 1993. The impact of sexual abuse on children: a review and synthesis of recent empirical literature. Psychological Bulletin 113: 164-180.

Krieger I 1974. Food restricton as a form of child abuse in ten cases of psychosocial deprivation dwarfism. Clinical Pediatrics 13: 127-133.

Layzer JI, Goodson BD \& DeLange C 1985. Children in shelters. Response 9: 2-5.

Levitt CJ 1993. Medical evaluation of the sexually abused child. Primary Care 20: 343-354.

Martin PH 1973. Nutrition: Its relationship to children's psysical, mental and emotional development. American Journal Clinical Nutrition 26: 775-776.

Martin PH, Beezley P, Conway EF \& Kempe H 1974. The development of abused children. Advances in Pediatrics 21: 25-73.

Martin SL, English KT, Clark KA, Cilenti D \& Kupper LL 1996. Violence and substance use among NorthCarolina pregnant women. American Journal of Public Health 86: 991-998.

McFarlane J, Parker B \& Soeken K 1996. Abuse during pregnancy: associations with maternal health and infant birth weight. Nursing Research 45: 37-42.

McRobbie A \& Thornton SL 1995. Rethinking moral panic for multi-mediated social worlds. British Journal of Sociology 46: 559-574.

Minayo MCS 1994. A violência social sob a perspectiva da saúde pública. Cadernos de Saúde Pública 10: 7-18.

Minayo MCS \& Souza ER 1998. Violência e saúde como um campo interdisciplinar e de ação coletiva. História, Ciências, Saúde IV: 513-531.

Núcleo de Pesquisa das Violências 1998. Violências Públicas e Privadas: Registros, Interpretações e Politicas. Projeto de Pesquisa. Instituto de Medicina Social/UERJ.

Parker B, Mc Farlane J \& Soeken K 1994. Abuse during pregnancy: effects on maternal complications and birth-weight in adult and teenage women. Obstetrics \& Gynecology 45: 37-42.

Pugh RJ 1978. The battered fetus. British Medical Journal 2: 858

Rappley M \& Speare KH 1993. Initial evaluation and interview techniques for child sexual abuse. Primary Care 20: 329-342. 
Reichenheim M \& Moraes C 1998. Alguns pilares para a apreciação da validade de estudos epidemiológicos. Revista Brasileira de Epidemiologia, no prelo.

Santos HO 1991. Crianças Violadas. CBIA/CRAMI, Brasília, $114 \mathrm{pp}$.

Schei B, Samuelsen SO \& Bakketeig LS 1991. Does spousal physical abuse affect the outcome of pregnancy? Scandinavian Journal of Social Medicine 19: 26-31.

Stewart DE \& Cecutti A 1993. Physical abuse in pregnancy. Canadian Medical Association Journal 149: 1257-1263.

Soares BM 1997. Mulheres Invisíveis: Violência Familiar e Formações Subjetivas. Tese de Doutorado, Instituto Universitário de Pesquisas do Rio de Janeiro, Rio de Janeiro, $328 \mathrm{pp}$.

Straus MA \& Gelles RJ 1995. Physical Violence in American Families. Risk Factors and Adaptations to Violence in 8,145 Families. Transaction Publishers, New Brunswick, London, 614 pp.
Straus MA, Gelles RJ \& Steinmetz S 1980. Behind Closed Doors. Anchor Press, New York.

Valdez-Santiago R \& Sanin-Aguirre LH 1996. La violencia doméstica durante el embarazo y su relación con el peso al nacer. Salud Pública de México 38: 352362.

Velho G 1996. Violência, reciprocidade e desigualdade: uma perspectiva antropológica, p. 10-24. In G Velho \& Alvite (orgs.) - Cidadania e Violência. Universidade Federal do Rio de Janeiro/Fundação Getúlio Vargas, Rio de Janeiro.

Viewiorka M 1997. O novo paradigma da violência. Tempo Social: Revista de Sociologia da USP 9: 5-41.

Widon CS 1989. Does violence beget violence? A critical examination of the literature. Psychological Bulletin 106: 3-28.

Witten CF, Petit MG \& Fischhoff J 1969. Evidence that growth failure from maternal deprivation is secondary to undereating. Journal of American Medical Association 209: 1675-1682. 